\title{
Tamanho e forma de parcela para pimentão em estufa plástica
}

\author{
Plot size and shape for chili pepper in plastic greenhouse
}

\author{
Leandro Homrich Lorentz ${ }^{I}$ Alessandro Dal’col Lúcio ${ }^{I I}$
}

\section{RESUMO}

O interior de uma estufa pode ser considerado um ambiente heterogêneo, o que se reflete numa grande variabilidade na produção, sendo necessário o uso de técnicas experimentais para a melhoria da precisão das inferências estatísticas. Dentre as formas de redução do erro experimental, está a busca do tamanho ótimo de parcela, que apresenta especial importância em experimentos com estufa plástica, visto que usualmente a área experimental é restrita às instalações já existentes. Este trabalho teve como objetivos: estimar o tamanho ótimo para a fitomassa fresca de frutos de pimentão cultivado em estufa plástica e relacionar os parâmetros das equações que determinam o tamanho ótimo de parcela. Conduziram-se quatro experimentos em branco sob cobertura plástica, cada um com oito fileiras de cultivo úteis de 70 plantas. Coletou-se em cada planta a fitomassa fresca dos frutos acumulada nas colheitas, identificando sua posição dentro da estufa pelo número da fileira e a sua posição dentro da fileira. Estimou-se o tamanho ótimo de parcela $\left(X_{0}\right)$ e se relacionaram estatísticas descritivas e os parâmetros usados para sua estimativa através do coeficiente de correlação de Pearson. Recomendou-se o uso de parcelas de 10 plantas na fileira ou fileiras duplas, com tendência a diminuir de tamanho com a permanência do experimento no campo. Não houve consistência na determinação das variáveis mais relacionadas com o tamanho ótimo da parcela.

Palavras-chave: Capsicum annuum L., precisão experimental, análise de trilha, índice de heterogeneidade da produção.

\section{ABSTRACT}

The interior of a plastic greenhouse can be considered a heterogeneous environment due high variability in the production, being necessary the use of experimental techniques for improvement of the statistical inferences precision. Between the main forms to obtain experimental error reduction is the use of the optimal plot size that has special importance in experiments inside plastic greenhouse, where the experimental area is normally restricted to the preexisting installations. The aim of this work is to estimate the optimal plot size for fruit fresh biomass of chili pepper cultivated in plastic greenhouse and the relation of the equations parameters that determine it. It was conducted four blank experiments under plastic covering, each one with eight lines of 70 plants. The fruit fresh biomass was evaluated in each plant, identifying its position inside the greenhouse, by the line number, and its position in the line in each harvest. The optimal plot size was estimated and related with the parameters used for its estimate by the Pearson correlation coefficient. It was recommended the use of parcels with ten plants in the line or double line, tending to decrease. There wasn't consistency in the relation of the variables with the plot size.

Key words: Capsicum annuum L., experimental precision, path analysis, yield heterogeneity index.

\section{INTRODUÇÃO}

A cultura do pimenteiro é de grande importância entre as plantas olerícolas, sendo bem adaptada ao cultivo protegido, constituindo-se em uma alternativa de renda para os pequenos produtores e para a agricultura familiar. O seu fruto é consumido em grande parte in natura, sendo também utilizado na indústria de processamento de alimentos devido à presença de pigmentos naturais na polpa, os quais são utilizados em corantes de sopas instantâneas e embutidos de carnes (REIFSCHNEIDER, 2000).

'Departamento de Zootecnia, Centro de Educação Superior do Oeste, Universidade do Estado de Santa Catarina (UDESC), 89802200, Chapecó, SC, Brasil. E-mail: leandrolorentz@yahoo.com.br. Autor para correspondência.

"Departamento de Fitotecnia, Centro de Ciências Rurais (CCR), Universidade Federal de Santa Maria (UFSM), Santa Maria, RS, Brasil. 
O cultivo em ambiente protegido pode favorecer a produção das culturas em comparação ao cultivo em campo, permitindo alongar o tempo de cultivo de uma cultura ou mesmo a antecipação na data de plantio, visto que aumenta o conforto térmico das plantas nos períodos frios. Além disso, esse tipo de cultivo as protege de chuvas intensas e excessivas, granizo e geadas, assim como permite um maior controle fitossanitário, o que provoca incrementos na produtividade e na qualidade do produto (SEGÓVIA et al., 1997; CUNHA \& ESCOBEDO, 2003).

Para que isso ocorra, são necessárias melhorias das características nutricionais e funcionais das culturas ou a geração de novas tecnologias envolvendo experimentos que permitam o controle do erro experimental para manter a precisão e a confiabilidade das inferências em um nível adequado. No caso do planejamento de experimentos com olerícolas em estufas plásticas, existe um efeito complicador, pois a área experimental é frequentemente limitada a instalações já existentes, além de haver uma fonte de variação adicional causada pelas sucessivas colheitas na mesma planta, somando-se isso ao constante manuseio das plantas devido aos tratos culturais intensivos. Para contornar essas dificuldades, é preciso que as técnicas de planejamento e a análise de dados sejam refinadas a fim de maximizar a qualidade da informação obtida, com menor dispêndio de espaço e com precisão compatível com a expectativa do pesquisador.

Dentre as técnicas reconhecidas pela literatura para a redução do erro experimental, descrito por STEEL et al. (1997) como a variação existente entre parcelas que receberam o mesmo tratamento, está a determinação do tamanho ótimo de parcela experimental. Nos experimentos em ambientes em área restrita, como dentro de uma estufa plástica, o tamanho de parcela é um parâmetro de grande importância e que deve ser otimizado, pois está intimamente relacionado ao número de repetições e de tratamentos que podem ser alocados na área experimental.

O tamanho de parcela estimado por um método algébrico, como os Métodos da Máxima Curvatura modificados por LESSMAN \& ATKINS (1963), MÉIER \& LESSMAN (1971) e THOMAS (1974), é o resultado da interação de diversos fatores que são quantificados na forma de relações empíricas, as quais indicam a variabilidade ambiental da área experimental. Se a magnitude do efeito desses fatores for compreendida de forma separada e conjunta, será possível direcionar esforços para minimizá-lo e aumentar a precisão dos resultados. Como já observado por STORCK et al. (1982) e VIANA et al. (2002), os diversos métodos de estimação do tamanho de parcela levam a resultados diferentes. Isso ocorre, principalmente, porque os métodos consideram de forma diferente as relações existentes entre as quantidades usadas na sua determinação.

Apesar de estarem disponíveis na literatura trabalhos que visaram a determinar o tamanho ótimo de parcela experimental (MELLO et al., 2004; OLIVEIRA et al., 2005), esses foram estimados utilizando-se apenas a produção total do cultivo avaliada em um único momento. Relatos quanto ao tamanho de parcela utilizando a produção em vários momentos, cuja soma constituirá a produção total, como no caso do cultivo de olerícolas, são raros na literatura. O mesmo ocorre com a persistência do índice de heterogeneidade da produção, quando são realizadas múltiplas colheitas em um mesmo cultivo. A estimativa do tamanho de parcela para cultivos protegidos foi realizada para pimenteiro (LÚCIO et al., 2004), abobrinha italiana (MELLO et al., 2004 e FEIJÓ et al., 2008) e tomateiro (LOPES et al., 1998). O estudo da relação entre dos coeficientes utilizados na estimação do tamanho ótimo de parcela por métodos algébricos e o tamanho estimado propriamente dito $\left(\mathrm{X}_{0}\right)$ foi realizado para milho (STORCK et al., 2006a) e batata (OLIVEIRA et al., 2006; STORCK et al., 2006b).

Este trabalho tem por objetivos estimar o tamanho ótimo de parcela para fitomassa fresca dos frutos de pimentão acumulada em sucessivas colheitas e correlacionar os parâmetros que o determinam.

\section{MATERIAL E MÉTODOS}

Foram conduzidos quatro experimentos com a cultura do pimentão em cultivo protegido durante os anos de 2001, 2002 e 2003, no Departamento de Fitotecnia da Universidade Federal de Santa Maria, nas coordenadas 2943'23”'S e 53\%43'15”'W e altitude de $95 \mathrm{~m}$. As sementes do híbrido Vidi foram adquiridas no comércio local e semeadas em bandejas de 128 alvéolos. As mudas foram transplantadas quando apresentavam de seis a oito folhas definitivas, ou aproximadamente $15 \mathrm{~cm}$ de estatura. O preparo do solo foi realizado com enxada rotativa, revolvendo-o antes e depois da distribuição dos fertilizantes, em dose equivalente a $130 \mathrm{~kg} \mathrm{ha}^{-1}$ de $\mathrm{N}, 70 \mathrm{~kg} \mathrm{ha}^{-1} \mathrm{P}_{2} \mathrm{O}_{5}$ e $230 \mathrm{~kg}$ $\mathrm{ha}^{-1}$ de $\mathrm{K}_{2} \mathrm{O}$. Na estação verão/outono $(\mathrm{V} / \mathrm{O})$, o transplante ocorreu em 13/02/2001 e 14/02/2002 (Experimentos 1 e 3, respectivamente), enquanto que, na estação primavera/verão (P/V), ocorreu em 28/09/ 2001 e 02/10/2003 (Experimentos 2 e 4, respectivamente). Foram realizadas cinco, quatro, oito e quatro colheitas, respectivamente, para os Experimentos 1, 2, 3 e 4. 
As estufas plásticas possuíam 24m de comprimento no sentido norte-sul e 10m de largura, pé direito de 2,0m e altura central de 3,5m, com estrutura de madeira na forma de arco pampeano, coberta com filme de PeBD 100micras, mesmo material utilizado nas portas e cortinas laterais. Aárea experimental no interior da estufa compôs-se de oito fileiras de cultivo com 70 plantas cada, orientadas no sentido longitudinal e espaçadas $0,30 \mathrm{~m}$ e $1,00 \mathrm{~m}$ entre os camalhões de $0,10 \mathrm{~m}$ de altura, cobertos com mulching opaco preto de PeBD 35 micras. As plantas foram irrigadas por tubos gotejadores instalados sob o mulching e deixadas em crescimento livre, com desbrotas periódicas nos ramos provenientes das axilas das folhas, suspensas por fios de ráfia presos a arames de metal na altura do pé-direito. $\mathrm{O}$ ponto de colheita se caracterizou pelo surgimento da coloração azulada na base do fruto. Coletou-se a fitomassa fresca dos frutos de pimentão acumulada nas colheitas de cada planta, anotando-se a fileira de cultivo e a posição da planta dentro da fileira, enquanto as plantas com produção nula nas colheitas receberam valor zero.

A partir da produção individual acumulada das plantas (unidade básica - UB), simularam-se parcelas de 15 tamanhos diferenciados, formando parcelas quadradas ou retangulares com maior comprimento na fileira de cultivo. Os tamanhos simulados foram: 1, 2, 5, 7, 10 e 14 plantas na fileira com uma fileira de largura; 2, 5, 7, 10 e 14, com duas fileiras de largura; 5, 7, 10 e 14 plantas na fileira com quatro fileiras de largura.

Para cada tamanho de parcela simulado, obteve-se: $\mathrm{X}$ : tamanho de parcela simulado em número de plantas; $\mathrm{J}_{\mathrm{x}}$ : número de repetições (560/X); $\mathrm{M}_{\mathrm{x}}$ : média da fitomassa fresca de frutos de pimentão das parcelas de tamanho $\mathrm{X} ; \mathrm{V}_{\mathrm{X}}$ : variância da fitomassa fresca das parcelas simuladas; $\mathrm{VU}_{\mathrm{x}}$ : variância reduzida de uma unidade básica, estimada por $\mathrm{VU}_{\mathrm{x}}=\mathrm{V}_{\mathrm{x}} / \mathrm{X}^{2} ; \mathrm{CV}_{\mathrm{x}}$ : coeficiente de variação das parcelas de $\mathrm{X}$ unidades básicas; $\mathrm{GL}_{\mathrm{x}}$, dado por $\mathrm{J}_{\mathrm{x}}-1$.

Estimou-se o índice de heterogeneidade da produção (b) por $\log \mathrm{VU}_{\mathrm{x}}-\log \mathrm{V}_{1}-\mathrm{b} \cdot \log \mathrm{X}$, em que $\mathrm{V}_{1}$ é a estimativa da variância entre as parcelas de uma unidade básica e ponderada pelos graus de liberdade associados aos respectivos tamanhos de parcela (STEEL et al., 1997), que variaram de 9 a 559. Analogamente à equação acima, estimou-se os coeficientes (A e B) da equação $\log \mathrm{CV}_{\mathrm{x}}-\log \mathrm{A}-\mathrm{B} \cdot \log$ $\mathrm{X}$, em que A estima o coeficiente de variação entre as parcelas de uma unidade básica. O tamanho de parcela para fitomassa fresca de pimentão $\left(\mathrm{X}_{0}\right)$, em número de plantas, foi estimado pelo método da Máxima Curvatura Modificado(MMCM), $\mathrm{X}_{0}=\left[\frac{\mathrm{A}^{2} \mathrm{~B}^{2}(2 \mathrm{~B}+1)}{\mathrm{B}+2}\right]^{\frac{1}{2 \mathrm{~B}+2}}$ (MÉIER \& LESSMAN, 1971).

A partir dos tamanhos de parcela obtidos, estimou-se a diferença verdadeira entre duas médias de tratamentos em porcentagem da média (d\%) para que ocorresse diferença significativa entre elas, dada por: $d \%=\sqrt{\frac{2\left(t_{1}+t_{2}\right)^{2} A^{2}}{J X_{0}^{b}}}$ (HATHEWAY, 1961), em que $t_{1}$ é o valor tabelado da distribuição t-Student para teste bilateral com 5\% de probabilidade de erro e $t_{2}$ é o valor tabelado da distribuição t-Student correspondente a 2(1-P) de probabilidade de se encontrar resultados significativos $(P=0,80)$, enquanto o valor $r$ indica $o$ número de blocos simulados $(\mathrm{J}=3,4,6$ e 8$)$ para $70 / \mathrm{X}_{0}$ tratamentos $(\mathrm{I})$, com $(\mathrm{I}-1)(\mathrm{J}-1)$ graus de liberdade.

Calculou-se para parcelas de uma planta o coeficiente de correlação de Pearson entre o número acumulado de colheitas realizadas (Colh), a média de produção para parcelas de uma planta $\left(\mathrm{M}_{1}\right)$, o desvio padrão $\left(\mathrm{S}_{1}\right)$, o coeficiente de variação $\left(\mathrm{CV}_{1}\right)$, os coeficientes $\mathrm{V}_{1}$, b, A e o tamanho ótimo de parcela $\left(\mathrm{X}_{0}\right)$ para cada colheita acumulada, dos quatro experimentos.

\section{RESULTADOS E DISCUSSÃO}

A tabela 1 contém o coeficiente de variação da fitomassa fresca acumulada de pimentão dos 15 diferentes tamanhos de parcelas simulados. Verificouse que, independentemente do tamanho, da forma e da estação sazonal, ocorreu decréscimo do coeficiente de variação com o acúmulo da produção nas colheitas, indicando ganho em precisão experimental com o prolongamento do experimento no campo. Isso pode ser observado, por exemplo, nas parcelas de cinco plantas por fileira no Experimento 1, cujo coeficiente de variação assumiu os valores de 52,6, 31,2, 21,1 20,8 e $18,1 \%$, ao acumular uma, duas, três, quatro e cinco colheitas, respectivamente. Da mesma forma, ocorreu uma redução do coeficiente de variação da fitomassa fresca total produzida com o aumento do número de parcelas na fileira $\left(\mathrm{X}_{1}\right)$, como visto no Experimento 1. Para a produção total acumulada nas colheitas (Colh = 5), ocorreu redução do CV de 38,0\% para 27,8, 18,1, $16,2,13,0$ e 12,0\% quando se aumentou de uma para duas, cinco, sete, 10 e 14 plantas por parcela, respectivamente (Tabela 1 ).

Entretanto, o aumento do tamanho da parcela em sua largura, pela utilização de duas ou quatro 
Tabela 1 - Número da colheita acumulada (Colh) e os respectivos coeficientes de variação em função do tamanho ( $\left.\mathrm{X}_{1 \mathrm{x}} \mathrm{X}_{2}\right)$ e da forma da parcela, dados pelo número de plantas na fileira de cultivo $\left(\mathrm{X}_{1}-\right.$ comprimento) e pelo número de fileiras $\left(\mathrm{X}_{2}-\right.$ largura $)$ para cada colheita acumulada. Santa Maria, 2008.

\begin{tabular}{|c|c|c|c|c|c|c|c|c|c|c|c|c|c|c|c|}
\hline Colh & $1 \mathrm{x} 1$ & $2 \mathrm{x} 1$ & $2 x 2$ & $5 \times 1$ & $5 \times 2$ & $5 \times 4$ & $7 \times 1$ & $7 x 2$ & $7 \times 4$ & 10x1 & $10 x 2$ & $10 \times 4$ & $14 x 1$ & $14 x 2$ & $14 \times 4$ \\
\hline & & & & & & & $\Gamma$ & - & & ton & & & & & ------ \\
\hline 1 & 88,0 & 65,2 & 47,8 & 52,6 & 40,7 & 31,8 & 48,5 & 37,0 & 31,4 & 44,7 & 35,7 & 30,2 & 40,7 & 30,8 & 27,6 \\
\hline 2 & 55,9 & 41,6 & 31,0 & 31,2 & 24,0 & 20,1 & 29,0 & 23,2 & 19,8 & 25,7 & 22,0 & 19,6 & 23,3 & 19,1 & 18,3 \\
\hline 3 & 42,4 & 31,5 & 22,1 & 21,1 & 14,3 & 10,5 & 19,2 & 13,3 & 10,0 & 15,1 & 11,9 & 9,8 & 13,2 & 10,2 & 8,6 \\
\hline 4 & 41,0 & 30,7 & 20,8 & 20,6 & 14,4 & 11,4 & 18,7 & 12,4 & 10,8 & 16,1 & 11,6 & 10,6 & 14,3 & 9,8 & 9,0 \\
\hline \multirow[t]{2}{*}{5} & 38,0 & 27,8 & 19,2 & 18,1 & 11,6 & 7,6 & 16,2 & 10,3 & 6,6 & 13,0 & 8,8 & 6,1 & 12,0 & 7,7 & 4,2 \\
\hline & & & & & Exper & nento 2 & Estaçẫ & sazona & primav & a/verão & 2001) & & & & \\
\hline 1 & 87,9 & 71,8 & 53,8 & 57,2 & 43,3 & 39,1 & 54,0 & 42,7 & 38,8 & 52,0 & 39,9 & 37,8 & 48,9 & 38,9 & 37,7 \\
\hline 2 & 57,6 & 41,3 & 28,9 & 27,4 & 17,1 & 14,2 & 23,7 & 16,2 & 13,7 & 21,3 & 13,3 & 11,6 & 18,9 & 13,3 & 12,5 \\
\hline 3 & 46,1 & 32,5 & 23,9 & 20,8 & 13,8 & 10,6 & 17,1 & 13,6 & 10,0 & 15,0 & 10,8 & 7,2 & 12,8 & 10,7 & 8,0 \\
\hline \multirow[t]{2}{*}{4} & 44,0 & 30,8 & 22,2 & 19,5 & 12,7 & 8,7 & 16,2 & 12,1 & 7,9 & 13,8 & 9,6 & 5,0 & 11,6 & 8,7 & 5,4 \\
\hline & & & & & -- Exp & imento & - Esta & o sazol & l verãc & utono & 002) -- & & & & ----- \\
\hline 1 & 150,9 & 121,0 & 76,9 & 91,6 & 59,0 & 41,3 & 86,5 & 53,5 & 39,1 & 78,4 & 47,8 & 35,8 & 77,1 & 46,2 & 33,5 \\
\hline 2 & 107,3 & 83,8 & 58,6 & 63,4 & 43,5 & 30,5 & 58,7 & 39,9 & 28,7 & 54,0 & 36,5 & 26,2 & 52,8 & 37,1 & 26,3 \\
\hline 3 & 81,8 & 63,5 & 44,9 & 48,5 & 35,9 & 27,3 & 44,3 & 32,5 & 26,6 & 41,1 & 31,2 & 25,9 & 38,6 & 29,1 & 23,9 \\
\hline 4 & 60,2 & 45,6 & 32,0 & 35,2 & 25,3 & 16,6 & 31,7 & 22,3 & 14,6 & 28,7 & 20,7 & 14,1 & 27,5 & 19,3 & 10,9 \\
\hline 5 & 53,9 & 39,9 & 27,5 & 31,4 & 21,1 & 14,1 & 28,0 & 18,0 & 11,8 & 25,9 & 16,7 & 12,2 & 23,9 & 15,4 & 8,7 \\
\hline 6 & 49,0 & 36,7 & 26,5 & 27,3 & 20,0 & 13,7 & 23,9 & 16,8 & 12,1 & 20,9 & 14,8 & 11,7 & 19,3 & 13,9 & 9,8 \\
\hline 7 & 46,9 & 35,0 & 25,4 & 25,1 & 19,1 & 13,1 & 21,3 & 16,0 & 11,3 & 19,1 & 13,7 & 10,6 & 16,9 & 12,2 & 7,9 \\
\hline \multirow[t]{2}{*}{8} & 45,5 & 33,0 & 23,9 & 23,5 & 18,0 & 12,7 & 18,8 & 13,9 & 10,0 & 17,4 & 12,3 & 9,9 & 14,9 & 10,6 & 7,5 \\
\hline & & & & & Expe & lento 4 & Estaçấ & sazon & primav & a/verão & 2003) & ----.- & & & ------ \\
\hline 1 & 96,3 & 73,6 & 51,9 & 54,2 & 38,8 & 27,8 & 50,2 & 34,1 & 24,8 & 42,4 & 29,0 & 21,1 & 40,9 & 27,6 & 20,8 \\
\hline 2 & 54,1 & 41,3 & 28,5 & 31,3 & 22,6 & 17,1 & 26,8 & 18,4 & 12,7 & 23,3 & 17,0 & 11,7 & 21,0 & 14,3 & 9,2 \\
\hline 3 & 44,6 & 35,0 & 25,8 & 26,7 & 20,8 & 15,2 & 23,7 & 17,5 & 11,0 & 21,4 & 17,1 & 11,3 & 19,3 & 14,9 & 7,8 \\
\hline 4 & 43,7 & 34,4 & 25,1 & 26,5 & 20,6 & 15,1 & 23,6 & 17,5 & 11,1 & 21,3 & 16,8 & 11,2 & 19,5 & 15,0 & 8,1 \\
\hline
\end{tabular}

fileiras de cultivo, gerou a redução do coeficiente de variação mais pronunciada para parcelas de mesmo tamanho da parcela na fileira. Isso pode ser observado nas parcelas de 10 plantas, pois o CV estimado ao fim de cinco colheitas (Experimento 1) foi de 11,6\% para $5 \times 2$ e $13,0 \%$ para 10x1. Da mesma forma, observou-se $10,3 \%$ para $7 \times 2$ e $12,0 \%$ para $14 \times 1$, mesmo comportamento verificado nos demais experimentos. O mesmo já havia sido detectado por MELLO et al. (2004), que recomendou uso de parcelas de 10 plantas e com duas plantas na fileira e cinco fileiras de largura, independentemente da estação sazonal.

A estimativa do tamanho ótimo de parcela para fitomassa fresca de pimentão acumulada e a diferença mínima significativa entre duas médias de tratamento (d\%) estão apresentadas na tabela 2. Nessa tabela, observou-se a inferioridade produtiva do Experimento 3 em relação aos demais. Nesse Experimento, apesar da realização de oito colheitas, produziu-se em média 702,2 gramas de fitomassa fresca de pimentão por planta ao fim do experimento. Já nos Experimentos 1, 2, e 4, produziu-se mais de um quilo de pimentões por planta, chegando a 1249,4g planta $^{-1}$ na estação primavera/verão de 2001. A produção inferior obtida no Experimento 3 justificou-se pelas condições climáticas desfavoráveis às plantas naquele cultivo, como alta temperatura e umidade relativa no interior da estufa plástica durante a floração, assim como por diversos dias de céu encoberto, o que não ocorreu com tal intensidade nos demais experimentos, pois a variedade utilizada, o manejo e os tratos culturais foram similares em todos os experimentos.

O índice de heterogeneidade da produção (b) apresentou um aumento no seu valor com a evolução das colheitas, chegando a valores superiores à unidade ao fim do experimento (Experimentos 1 e 2), ou nas colheitas intermediárias (Experimentos 3 e 4), indicando alta variabilidade da produção com a realização das colheitas. Além disso, os valores de $b=1,0$ indicam ocorrência de competição por recursos entre as plantas dentro da parcela (THOMAS, 1974). LÚCIO et al. (2004) observaram índices de heterogeneidade da produção para a cultura de pimentão entre 0,721 e 0,994, conforme a estação de cultivo, enquanto FEIJÓ et al. (2008) obtiveram valores de b entre 0,025 e 0,888 em produções acumuladas em 27 colheitas de abobrinha italiana. Para 
Tabela 2 - Número da colheita acumulada (Colh), fitomassa fresca média por planta acumulada nas colheitas (g.planta ${ }^{-1}$, $\left.\mathrm{M}_{1}\right)$, percentagem da fitomassa fresca dos frutos (Perc), coeficiente de variação $\left(C_{1}\right)$, estimativa dos parâmetros das equações $V_{(X)}=V_{1} / X^{b}$ e $\mathrm{CV}_{(\mathrm{X})}=\mathrm{A} / \mathrm{X}^{\mathrm{B}}$, tamanho ótimo de parcela $\left(\mathrm{X}_{0}\right)$ e diferença mínima significativa entre tratamentos (d\%) para 3, 4, 6 e 8 repetições simuladas. Santa Maria, 2008.

\begin{tabular}{|c|c|c|c|c|c|c|c|c|c|c|c|c|c|}
\hline \multirow{2}{*}{ Colh } & \multirow{2}{*}{$\mathrm{M}_{1}$} & \multirow{2}{*}{ Perc } & \multirow{2}{*}{$\mathrm{CV}_{1}$} & \multirow{2}{*}{$\mathrm{V}_{1}$} & \multirow{2}{*}{$\mathrm{b}$} & \multirow{2}{*}{ A } & \multirow{2}{*}{$\mathrm{B}$} & \multirow{2}{*}{$\mathrm{X}_{0}$} & \multirow{2}{*}{$\mathrm{I}^{1}$} & \multicolumn{4}{|c|}{--------------- Repetições ------------- } \\
\hline & & & & & & & & & & 3 & 4 & 6 & 8 \\
\hline & --------- & $x^{2}$ & 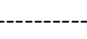 & $-----\mathrm{E}$ & erimentc & - Estac & sazonal & ão/o & $(200$ & ---------- & --------- & -------- & -------- \\
\hline 1 & 213,3 & 20,9 & 88,0 & 0,025 & 0,503 & 74,5 & 0,252 & 9 & 7 & 106,76 & 89,78 & 71,65 & 61,45 \\
\hline 2 & 476,5 & 46,6 & 55,9 & 0,038 & 0,413 & 40,7 & 0,207 & 5 & 14 & 69,31 & 59,25 & 47,88 & 41,29 \\
\hline 3 & 709,2 & 69,4 & 42,4 & 0,057 & 0,697 & 33,8 & 0,348 & 6 & 11 & 43,50 & 37,04 & 29,84 & 25,69 \\
\hline 4 & 860,3 & 84,2 & 41,0 & 0,074 & 0,635 & 31,6 & 0,317 & 5 & 14 & 45,06 & 38,52 & 31,13 & 26,84 \\
\hline \multirow[t]{2}{*}{5} & 1021,4 & 100,0 & 38,0 & 0,251 & 1,179 & 49,0 & 0,589 & 8 & 8 & 35,37 & 29,88 & 23,93 & 20,55 \\
\hline & & & & ---- Exp & imento & Estaçã & azonal $\mathrm{p}$ & aver & o $(2$ & ) -------- & & & ------- \\
\hline 1 & 437,6 & 34,6 & 87,9 & 0,091 & 0,325 & 69,0 & 0,163 & 6 & 11 & 123,99 & 105,56 & 85,04 & 73,23 \\
\hline 2 & 756,7 & 59,9 & 57,6 & 0,092 & 0,636 & 40,1 & 0,318 & 6 & 11 & 54,54 & 46,43 & 37,41 & 32,21 \\
\hline 3 & 1123,8 & 89,0 & 46,1 & 0,186 & 0,821 & 38,4 & 0,410 & 6 & 11 & 44,27 & 37,69 & 30,36 & 26,15 \\
\hline \multirow[t]{2}{*}{4} & 1263,1 & 100,0 & 44,0 & 0,399 & 1,135 & 50,1 & 0,568 & 8 & 8 & 37,86 & 31,98 & 25,61 & 22,00 \\
\hline & & & & ----- Ex & erimentc & - Estaç & sazonal & ão/o & $(200$ & - & - & & ------- \\
\hline 1 & 68,5 & 9,8 & 150,9 & 0,013 & 0,822 & 169,1 & 0,411 & 18 & 3 & 156,35 & 122,11 & 92,41 & 77,60 \\
\hline 2 & 126,7 & 18,0 & 107,3 & 0,020 & 0,741 & 111,0 & 0,370 & 13 & 5 & 111,96 & 92,61 & 73,00 & 62,30 \\
\hline 3 & 207,6 & 29,6 & 81,7 & 0,022 & 0,560 & 72,0 & 0,280 & 9 & 7 & 96,97 & 81,55 & 65,08 & 55,82 \\
\hline 4 & 366,1 & 52,1 & 60,2 & 0,084 & 0,953 & 79,1 & 0,476 & 11 & 6 & 64,02 & 53,49 & 42,48 & 36,37 \\
\hline 5 & 448,1 & 63,8 & 53,8 & 0,104 & 1,011 & 71,9 & 0,505 & 10 & 7 & 55,97 & 47,07 & 37,56 & 32,22 \\
\hline 6 & 557,5 & 79,4 & 49,0 & 0,080 & 0,815 & 50,8 & 0,407 & 8 & 8 & 53,60 & 45,27 & 36,25 & 31,14 \\
\hline 7 & 608,7 & 86,7 & 46,9 & 0,108 & 0,926 & 54,1 & 0,463 & 8 & 8 & 50,86 & 42,96 & 34,40 & 29,55 \\
\hline \multirow[t]{2}{*}{8} & 702,2 & 100,0 & 45,5 & 0,112 & 0,903 & 47,8 & 0,452 & 8 & 8 & 45,93 & 38,80 & 31,07 & 26,69 \\
\hline & & & & --- Exp & imento & Estaçã & azonal $\mathrm{p}$ & aver & o (2) & ) --------- & & & ------- \\
\hline 1 & 269,3 & 23,9 & 96,3 & 0,074 & 0,808 & 100,9 & 0,404 & 13 & 5 & 93,39 & 77,25 & 60,89 & 51,96 \\
\hline 2 & 702,1 & 62,3 & 54,1 & 0,238 & 0,979 & 69,4 & 0,490 & 10 & 7 & 56,04 & 47,12 & 37,61 & 32,26 \\
\hline 3 & 1059,8 & 94,0 & 44,6 & 0,494 & 1,004 & 66,4 & 0,502 & 10 & 7 & 52,05 & 43,77 & 34,93 & 29,96 \\
\hline 4 & 1127,2 & 100,0 & 43,7 & 0,509 & 0,972 & 63,2 & 0,486 & 9 & 7 & 54,20 & 45,58 & 36,38 & 31,20 \\
\hline
\end{tabular}

${ }^{1}$ : Número de tratamentos $=70 / \mathrm{X}_{0}$.

essa mesma cultura, MELLO et al. (2004) observou valores de b de 0,74 e 0,97 , que pode ser pode ser traduzido como elevada variabilidade ambiental no interior de uma estufa plástica com plantas cultivadas no solo. Já o tamanho estimado da parcela na maioria dos casos reduziu-se com o acúmulo da produção das colheitas, estabilizando-se entre 8 e 10 plantas a partir de aproximadamente $50 \%$ da produção colhida.

O aumento da precisão com a manutenção do experimento no campo se confirmou também devido aos valores de $\mathrm{d} \%$, que em geral foram decrescentes com o acúmulo da produção das colheitas. Ou seja, em experimentos mais duradouros, nos quais são realizadas mais colheitas e se acumula maior volume de produção, existe maior probabilidade de serem encontradas diferenças significativas entre dois tratamentos até as últimas colheitas. Conforme observado no Experimento 1, se for planejado um experimento com oito tratamentos e quatro repetições, será necessária uma diferença superior a 29,88\% entre as médias, para que sejam consideradas significativamente diferentes, caso sejam realizadas cinco colheitas (Tabela 2). Os valores de d\% também se reduziram com o aumento do número de repetições por tratamento, como no exemplo anterior, que caso se utilizar oito repetições ao invés de quatro, o valor de d\% passará a ser 20,55\%. Nesses casos, ocorre um aumento gradativo nos graus de liberdade, associado ao erro e à obtenção de uma estimativa fidedigna da variação residual.

Na tabela 3, observa-se que as variáveis que subentendem a duração do experimento no campo, número de colheitas (Colh) e média da colheita $\left(\mathrm{M}_{1}\right)$, foram inversamente relacionadas ao coeficiente de variação (CV), com valores médios do coeficiente de correlação (r) a 0,983 e -0,888, respectivamente. O fato de os valores de CV decrescerem com o acúmulo da produção nas colheitas já era esperado, pois quanto 
Tabela 3 - Coeficientes de correlação estimados para cada experimento (Exp) entre estatísticas descritivas e as características determinantes do tamanho de parcela. Santa Maria, 2008.

\begin{tabular}{|c|c|c|c|c|c|c|c|c|}
\hline Exp & Variável & $\mathrm{M}_{1}$ & $\mathrm{~S}_{1}$ & $\mathrm{CV}_{1}$ & $\mathrm{~V}_{1}$ & b & A & Хo \\
\hline $1^{1}$ & \multirow{5}{*}{ Colheita } & 0,992* & 0,988* & $-0,878^{\mathrm{NS}}$ & $0,835^{\mathrm{NS}}$ & $0,837^{\mathrm{NS}}$ & $-0,547^{\mathrm{NS}}$ & $-0,174^{\mathrm{NS}}$ \\
\hline $2^{2}$ & & $0,986 *$ & $0,990 *$ & $-0,914^{*}$ & $0,906 *$ & $0,995 *$ & $-0,537^{\mathrm{NS}}$ & $0,775^{\mathrm{NS}}$ \\
\hline $3^{3}$ & & $0,994 *$ & $0,992 *$ & $-0,890^{*}$ & $0,911 *$ & $0,472^{\mathrm{NS}}$ & $-0,864^{*}$ & $-0,834^{*}$ \\
\hline $4^{4}$ & & $0,961^{*}$ & $0,960^{*}$ & $-0,869^{\mathrm{NS}}$ & $0,957^{*}$ & $0,743^{\mathrm{NS}}$ & $-0,858^{\mathrm{NS}}$ & $-0,894^{\mathrm{NS}}$ \\
\hline Média & & 0,983 & 0,983 & $-0,888$ & 0,902 & 0,762 & $-0,702$ & $-0,282$ \\
\hline 1 & \multirow{5}{*}{$\mathrm{M}_{1}$} & & $0,994 *$ & $-0,929^{\mathrm{NS}}$ & $0,780^{\mathrm{NS}}$ & $0,792^{\mathrm{NS}}$ & $-0,639^{\mathrm{NS}}$ & $-0,269^{\mathrm{NS}}$ \\
\hline 2 & & & $0,996 *$ & $-0,951^{*}$ & $0,832^{\mathrm{NS}}$ & $0,971^{*}$ & $-0,633^{\mathrm{NS}}$ & $0,658^{\mathrm{NS}}$ \\
\hline 3 & & & $0,996 *$ & $-0,891^{*}$ & $0,933^{*}$ & $0,532^{\mathrm{NS}}$ & $-0,847^{*}$ & $-0,808 *$ \\
\hline 4 & & & $0,999 *$ & $-0,954^{*}$ & $0,989 *$ & $0,881^{\text {NS }}$ & $-0,935^{\mathrm{NS}}$ & $-0,928^{\mathrm{NS}}$ \\
\hline Média & & & 0,996 & $-0,931$ & 0,884 & 0,794 & $-0,764$ & $-0,337$ \\
\hline 1 & \multirow{5}{*}{$\mathrm{S}_{1}$} & & & $-0,929^{\mathrm{NS}}$ & $0,776^{\mathrm{NS}}$ & $0,760^{\mathrm{NS}}$ & $-0,652^{\mathrm{NS}}$ & $-0,314^{\mathrm{NS}}$ \\
\hline 2 & & & & $-0,920 *$ & $0,871^{\mathrm{NS}}$ & $0,973 *$ & $-0,562^{\mathrm{NS}}$ & $0,702 *$ \\
\hline 3 & & & & $-0,926^{*}$ & $0,933^{*}$ & $0,508^{\mathrm{NS}}$ & $-0,886^{*}$ & $-0,847 *$ \\
\hline 4 & & & & $-0,959 *$ & $0,986^{*}$ & $0,886^{\mathrm{NS}}$ & $-0,941^{\mathrm{NS}}$ & $-0,934^{\mathrm{NS}}$ \\
\hline Média & & & & $-0,934$ & 0,892 & 0,782 & $-0,760$ & $-0,348$ \\
\hline 1 & \multirow{5}{*}{$\mathrm{CV}_{1}$} & & & & $-0,562^{\mathrm{NS}}$ & $-0,573^{\mathrm{NS}}$ & $0,861^{\mathrm{NS}}$ & $0,557^{\mathrm{NS}}$ \\
\hline 2 & & & & & $-0,660 *$ & $-0,915^{*}$ & $0,833^{\mathrm{NS}}$ & $-0,493^{\mathrm{NS}}$ \\
\hline 3 & & & & & $-0,874 *$ & $-0,409^{\mathrm{NS}}$ & $0,969 *$ & $0,938 *$ \\
\hline 4 & & & & & $-0,904^{\mathrm{NS}}$ & $-0,976 *$ & $0,996 *$ & $0,974 *$ \\
\hline Média & & & & & $-0,750$ & $-0,718$ & 0,915 & 0,494 \\
\hline 1 & \multirow{5}{*}{$\mathrm{V}_{1}$} & & & & & $0,964^{*}$ & $-0,071^{\mathrm{NS}}$ & $0,296^{\mathrm{NS}}$ \\
\hline 2 & & & & & & $0,905^{*}$ & $-0,137^{\mathrm{NS}}$ & $0,952 *$ \\
\hline 3 & & & & & & $0,762 *$ & $-0,754^{*}$ & $-0,692^{\mathrm{NS}}$ \\
\hline 4 & & & & & & $0,819^{\mathrm{NS}}$ & $-0,874^{\mathrm{NS}}$ & $-0,863^{\mathrm{NS}}$ \\
\hline Média & & & & & & 0,863 & $-0,459$ & $-0,077$ \\
\hline 1 & \multirow{5}{*}{ b } & & & & & & $-0,081^{\mathrm{NS}}$ & $0,353^{\mathrm{NS}}$ \\
\hline 2 & & & & & & & $-0,544^{\mathrm{NS}}$ & $0,798^{\mathrm{NS}}$ \\
\hline 3 & & & & & & & $-0,182^{\text {NS }}$ & $-0,086^{\mathrm{NS}}$ \\
\hline 4 & & & & & & & $-0,972^{*}$ & $-0,922^{\mathrm{NS}}$ \\
\hline Média & & & & & & & $-0,445$ & 0,036 \\
\hline 1 & \multirow{5}{*}{ A } & & & & & & & $0,884^{\mathrm{NS}}$ \\
\hline 2 & & & & & & & & $0,033^{\mathrm{NS}}$ \\
\hline 3 & & & & & & & & $0,993 *$ \\
\hline 4 & & & & & & & & $0,986 *$ \\
\hline Média & & & & & & & & 0,724 \\
\hline
\end{tabular}

1: $n=4,2: n=5,3: n=8,4: n=4 ; M_{1}$ : média da fitomassa fresca, $S_{1}$ : desvio padrão, $\mathrm{CV}_{1}$ : coeficiente de variação, $V_{1}$ : variância estimada para parcelas de uma unidade básica, b: índice de heterogeneidade da produção, A: coeficiente de variação estimado para parcelas de uma unidade básica, Xo: tamanho ótimo de parcela estimado para produção acumulada nas colheitas; *: significativo pelo teste t a 5\% de probabilidade de erro; ${ }^{\text {NS}}$ : não significativo.

mais plantas na fileira de cultivo possuírem frutos aptos a serem colhidos, menor será o número de plantas nas parcelas e nas fileiras com produção nula, o que implica, por consequência, o aumento da média da produção da parcela. Ainda assim, os valores de CV podem ser considerados como médios ou altos, mesmo para produção total, com valores entre 38,0 e 45,5\%, conforme a estação de cultivo (Tabela 2).

$\mathrm{O}$ índice de heterogeneidade da produção (b) foi positivamente correlacionado em todos os experimentos com essas variáveis, indicando que a correlação entre as parcelas adjacentes diminui com a 
realização de sucessivas colheitas. LOPES et al. (1998), que também observaram valores crescentes de b nas sucessivas colheitas, atribuíram esse fato a danos por injúrias causadas nas plantas por tratos culturais e colheita, a falhas na irrigação, a pequenas diferenças na fertilidade e à compactação do solo, que impedem o crescimento radicular. Além disso, atribuíram o mesmo fato ainda à competição entre plantas vizinhas por recursos como luz, água e nutrientes, que se torna mais evidente com a permanência das plantas no campo. Entretanto, esse comportamento se diferenciou do resultado apresentado por FEIJÓ et al. (2008), que não observaram tendência crescente dos valores de b com as sucessivas colheitas nas plantas de abobrinha italiana.

Observou-se inconsistência na correlação entre o $\mathrm{X}_{0}$ e b e $\mathrm{X}_{0}$ e $\mathrm{A}$, pois geraram valores próximos a zero e a unidade, não sendo possível a indicação segura do relacionamento entre eles. Esse comportamento observado é contrário ao esperado, pois STORCK et al. (1982) citaram que o índice de heterogeneidade da produção (b) é a principal variável explicativa do tamanho de parcela.

A relação do tamanho de parcela $\left(\mathrm{X}_{0}\right)$ com as outras variáveis no Experimento 2 apresentou um comportamento distinto dos outros Experimentos, seja pelo sinal, seja pela magnitude da correlação (Tabela 3). Esses resultados podem ter sido influenciados pelo tempo de duração do experimento, pois esse teve duração de 68 dias entre o transplante e a quarta colheita, com 47 dias para a primeira colheita, enquanto os demais experimentos foram colhidos pela primeira vez aos 65,56 e 82 dias, apresentarando duração total de 128, 126 e 102 dias, respectivamente (Experimentos $1,3$ e 4$)$.

\section{CONCLUSÕES}

O tamanho ótimo da parcela para fitomassa fresca de pimentão cultivado em estufa plástica é de 10 plantas, com cinco plantas na fileira e duas fileiras de largura. Não se encontrou consistência na indicação das variáveis mais importantes na determinação do tamanho ótimo da parcela.

\section{AGRADECIMENTOS}

Ao Conselho Nacional De Desenvolvimento Científico E Tecnológico (CNPQq), pelo Auxílio Financeiro e Pela Concessão de Bolsa de Produtividade em Pesquisa para Alessandro Dal'col Lúcio.

\section{REFERÊNCIAS}

CUNHA, A.R.; ESCOBEDO, J.F. Alterações micrometeorológicas causadas pela estufa plástica e seus efeitos no crescimento e produção da cultura do pimentão. Revista Brasileira de Agrometeorologia, v.11, n.1, p.15-26, 2003.

FEIJÓ, S. et al. Heterogeneity index of zucchini yields on protected environmental and experimental planning. Horticultura Brasileira, v.26, p.35-39, 2008. Disponível em: <http://www.scielo.br/pdf/hb/v26n1/a07v26n1.pdf>. Acesso em: 15 out. 2008. doi: 10.1590/S0102-05362008000100007.

HATHEWAY, W.H. Convenient plot size. Agronomy Journal, v.53, p.279-280, 1961.

LESSMAN, K.J.; ATKINS, R.E. Optimum plot size and relative efficiency of lattice designs for grain sorghum yield tests. Crop Science, v.3, p.477-841, 1963.

LOPES, S.J. et al. Técnicas experimentais para tomateiro tipo salada sob estufas plásticas. Ciência Rural, v.28, n.2, p.193197, 1998. Disponível em: <http://www.scielo.br/pdf/cr/v28n2/ a02v28n2.pdf $>$. Acesso em: 10 out. 2008. doi: 10.1590/ S0103-84781998000200002.

LÚCIO, A.D. et al. Estimativa de parâmetros para planejamento de experimentos com a cultura do pimentão em área restrita. Horticultura Brasileira, v.22, n.4, p.766-770, 2004. Disponível em: <http://www.scielo.br/pdf/hb/v22n4/23192.pdf>. Acesso em: 10 out. 2008. doi: 10.1590/S010205362004000400020 .

MÉIER, V.D.; LESSMAN, K.J. Estimation of optimum field plot shape and size for testing yield in Crambe abssinica. Crop Science, v.11, p.648-650, 1971.

MELLO, R.M. et al. Size and form of plots for the culture of the italian punpkin in plastic greenhouse. Scientia Agricola, v.61, n.4, p.457-461, 2004. Disponível em: <http:// www.scielo.br/pdf/sa/v61n4/22165.pdf $>$. Acesso em: 10 out. 2008. doi:10.1590/S0103-90162004000400017.

OLIVEIRA, S.J.R. et al. Plot size and experimental unit relationship in explanatory experiments. Scientia Agricola, v.62, n.6, p.585-589, 2005. Disponível em: <http:// www.scielo.br/pdf/sa/v62n6/a12v62n6.pdf>. Acesso em: 10 out. 2008. doi: 10.1590/S0103-90162005000600012.

OLIVEIRA, S.J.R. et al. Índice de heterogeneidade, coeficiente de variação e tamanho ótimo de parcela em batata. Ciência Rural, v.36, n.6, p.1710-1716, 2006. Disponível em: <http:/ /www.scielo.br/pdf/cr/v36n6/a07v36n6.pdf>. Acesso em: 10 out. 2008. doi: 10.1590/S0103-84782006000600007.

REIFSCHNEIDER, F.J.B. Capsicum: pimentas e pimentões no Brasil. Brasília: EMBRAPA, 2000. 113p.

SEGÓVIA, J.F.O. et al. Comparação do crescimento e desenvolvimento da alface no interior e no exterior de uma estufa de polietileno em Santa Maria, RS. Ciência Rural, v.27, n.1, p.37-41, 1997. Disponível em: < http://www.scielo.br/ pdf/cr/v27n1/a07v27n1.pdf>. Acesso em: 10 out. 2008. doi: 10.1590/S0103-84781997000100007. 
STEEL, R.G.D. et al. Principles and procedures of statistics: a biometrical approach. New York: McGraw-Hill, 1997. $666 \mathrm{p}$

STORCK, L. et al. Comparação de métodos de estimativa do índice de heterogeneidade do solo e do tamanho ótimo de parcela em experimentos com soja. Revista do Centro de Ciências Rurais, v.12, n.2-3, p.189-202, 1982.

STORCK, L. et al. Tamanho ótimo de parcela em experimentos com milho relacionado a metodologias. Revista Brasileira de Milho e Sorgo, v.5, n.1, p.48-57, 2006a. Disponível em: <http://www.abms.org.br/revista/revista_v5_n1/PDF/ E_ARTIGO_LINDOLFO.pdf>. Acesso em: 10 out. 2008.
STORCK, L. et al. Dimensões dos ensaios e estimativas do tamanho de parcela em batata. Pesquisa Agropecuária Brasileira, v.41, n.6, p.903-909, 2006b. Disponível em: $<$ http://www.scielo.br/pdf/pab/v41n6/30853.pdf >. Acesso em: 10 out. 2008. doi: 10.1590/S0100-204X2006000600002.

THOMAS, E.J. Relationship between plot size and plot variance. Agriculture Research Journal of Kerala, v.12, n.2, p.178189, 1974

VIANA, A.E.S. et al. Estimation of optimum plot sizes in field experiments with annatto. Bragantia, v.61, n.2, p.181-185, 2002. Disponível em: <http://www.scielo.br/pdf/brag/v61n2/ 18478.pdf>. Acesso em: 10 out. 2008. doi: 10.1590/S000687052002000200011 . 